\title{
How to Realize One-dimensional Discrete-time Quantum Walk by Dirac Particle
}

\author{
Yutaka SHIKANO $1,2,3, *$ \\ ${ }^{1}$ Institute for Molecular Science, Natural Institutes of Natural Sciences, \\ 38 Nishigo-Naka, Myodaiji, Okazaki, Aichi 444-8585, Japan \\ ${ }^{2}$ Institute for Quantum Studies, Chapman University, 1 University Dr., Orange, California 92866, USA \\ ${ }^{3}$ Materials and Structures Laboratory, Tokyo Institute of Technology, 4259 Nagatsuta, Yokohama 226-8503, Japan
}

\begin{abstract}
One-dimensional discrete-time quantum walks (DTQWs) can simulate various quantum and classical dynamics and have already been implemented in several physical systems. This implementation needs a well-controlled quantum dynamical system, which is the same requirement for implementing quantum information processing tasks. Here, we consider how to realize DTQWs by Dirac particles toward a solid-state implementation of DTQWs.
\end{abstract}

KEYWORDS: discrete-time quantum walk, Dirac particle, physical implementation

\section{Introduction}

The discrete-time quantum walk (DTQW) is defined as the quantum-mechanical analogue of the discrete-time random walk and has many applications, especially in quantum information science. Recent progress on quantum complexity has been based on the quantum walk [1,2], and other applications and insights have been reported in recent review papers [3-6] and books [7, 8]. It is remarked that the brief history of the DTQW was proposed by Feynman as the Feynman checkerboard [9] and thereafter independently formulated in quantum probability [10], quantum random walks [11], and quantum cellular automaton [12].

Owing to the developed quantum technology, there are several physical implementations [13-22]. However, it is emphasized that there is no implementation of the DTQW in a solid-state system. The simplest implementation in a photonic system is to use a polarized beam splitter or shifter and polarizers. Other experimental proposals of DTQWs have been demonstrated for an ion trap [23], cavity quantum electrodynamics [24], a non-Abelian anyon [25], BoseEinstein condensation [26], angular momentum classical light [27], ensembles of nitrogen-vacancy centers in diamond coupled to a superconducting qubit [28], and an optomechanical system [29].

Various DTQWs can simulate the Dirac equation [30-34], the spatially discretized Schrödinger equation [35, 36], the Klein-Gordon equation [33,37], or other various differential equations [38,39]. Furthermore, classical dynamics can be simulated [40]. On the other hand, we have not yet shown how to realize the DTQW in natural classical/quantum dynamics. In this paper, we will show that the massive Dirac equation naturally has the structure of the DTQW by the discretization of the space and time.

\section{Review of the Discrete-Time Quantum Walk}

Let us recapitulate the discrete-time random walk. First, we prepare a particle, which is located at the origin at the beginning, and a coin. We repeatedly carry out the following procedures:

(1) A coin flip,

(2) A shift in the particle depending on whether the coin is tails or heads.

Finally, we calculate the probability distribution $\operatorname{Pr}(n ; t)$ at the position $n$ and step $t$.

Analogous to the discrete-time random walk, we define the DTQW [41]. First, we prepare the quantum particle, which is located in the origin at the beginning and is labeled with a position state denoted by $|0\rangle$, and the quantum coin with an orthogonal basis: $|\uparrow\rangle=(1,0)^{\mathbf{T}}$ and $|\downarrow\rangle=(0,1)^{\mathbf{T}}$, where $\mathbf{T}$ is the transpose operator. We repeatedly carry out the following procedures:

This work is supported by the DAIKO Foundation. Also, this work was supported in part by Perimeter Institute for Theoretical Physics. Research at Perimeter Institute is supported by the Government of Canada through Industry Canada by the Providence of Ontario through the Ministry of Economic Development \& Innovation.

*Corresponding author. E-mail: yshikano@ims.ac.jp; shikano@chapman.edu 
(1) A quantum coin flip: This operator is generally defined as

$$
\begin{aligned}
C_{t} & =\sum_{n}\left[( a _ { n , t } | n , \uparrow \rangle + c _ { n , t } | n , \downarrow \rangle ) \left\langlen, \uparrow\left|+\left(d_{n, t}|n, \downarrow\rangle+b_{n, t}|n, \uparrow\rangle\right)\langle n, \downarrow|\right]\right.\right. \\
& =\sum_{n}\left[|n\rangle\langle n| \otimes\left(\begin{array}{ll}
a_{n, t} & b_{n, t} \\
c_{n, t} & d_{n, t}
\end{array}\right)\right]=: \sum_{n}\left[|n\rangle\langle n| \otimes \hat{C}_{n, t}\right],
\end{aligned}
$$

where $\hat{C}_{n, t} \in U(2)$, and $\hat{C}_{n, t}$ expresses the coin operator at the position $n$ and step $t$.

(2) A shift depending on whether the coin is tails or heads: This operator is defined as

$$
W=\sum_{n}(|n-1, \uparrow\rangle\langle n, \uparrow|+| n+1, \downarrow\rangle\langle n, \downarrow|) .
$$

It is noted that these procedures should be described as unitary processes. One step of the quantum walk operator at the step $t$ is defined as $U_{t}=W C_{t}$. This representation is focused on the quantum coin.

On the other hand, as the quantum-particle representation, we define the quantum-particle state as

$$
\Psi(n, t) \equiv e_{n, t}|\uparrow\rangle+f_{n, t}|\downarrow\rangle
$$

and its dynamics as

$$
\Psi(n, t+1)=P_{n, t} \Psi(n+1, t)+Q_{n, t} \Psi(n-1, t)
$$

with

$$
P_{n, t}+Q_{n, t}=C_{n, t} .
$$

This representation is a good fit to the combinatorial approach of the DTQW to first show the weak limit theorem $[42,43]$.

As alluded to in the introduction, various DTQWs can simulate several dynamics. For example, the connection to the massive Dirac equation can be seen in the following theorem.

Theorem 2.1 (Strauch [31]). The quantum coin is set as

$$
C(\epsilon)=\left(\begin{array}{cc}
\cos \epsilon & -i \sin \epsilon \\
-i \sin \epsilon & \cos \epsilon
\end{array}\right) .
$$

When the parameter $\epsilon$ is sufficiently small, this DTQW can simulate the massive Dirac equation.

This analysis is similar to Ref. 30 and can be extended to the two-dimensional space [32]. It is emphasized that the DTQW can be implemented under well-controlled nonrelativistic quantum mechanics while the DTQW can simulate the relativistic quantum mechanics. This originates from the discrete structure of space and time in the DTQW. Therefore, DTQWs can simulate several quantum and classical dynamics to unify the concept as a quantum dynamical simulator [6]. However, there remains the question of whether or not the relativistic particle can experimentally implement several DTQWs. In the following section, we discuss this question.

\section{DTQW Implementation by Dirac Particle}

Let us consider the massive Dirac equation as

$$
\partial_{t} \varphi(x, t)=-i\left(m \sigma_{x}+\sigma_{z} \partial_{x}\right) \varphi(x, t),
$$

where $\varphi(x, t)$ represents the Dirac spinor. By the Lax method, we derive the discretized equation as

$$
\begin{aligned}
& \frac{\varphi(x, t+\Delta t)-\varphi(x, t)}{\Delta t} \\
& \quad=-i\left(m \sigma_{x} \frac{\varphi(x+\Delta x, t)+\varphi(x-\Delta x, t)}{2}+\sigma_{z} \frac{\varphi(x+\Delta x, t)-\varphi(x-\Delta x, t)}{2 \Delta x}\right)+\mathcal{O}\left((\Delta t)^{2},(\Delta x)^{2}\right) .
\end{aligned}
$$

To compare the discretized Dirac equation with the quantum-walk dynamics in (2.4), the quantum state can be rewritten as

$$
\begin{aligned}
\varphi(x, t+\Delta t)= & \left(1-i \Delta t m \sigma_{x}\right) \frac{\varphi(x+\Delta x, t)+\varphi(x-\Delta x, t)}{2} \\
& -\frac{i}{2} \frac{\Delta t}{\Delta x} \sigma_{z}(\varphi(x+\Delta x, t)-\varphi(x-\Delta x, t))+\mathcal{O}\left((\Delta t)^{3},(\Delta x)^{2}\right) \\
\simeq & \frac{1}{2}\left(1-i \Delta t m \sigma_{x}-i \frac{\Delta t}{\Delta x} \sigma_{x}\right) \varphi(x+\Delta x, t)
\end{aligned}
$$




$$
\begin{aligned}
& +\frac{1}{2}\left(1-i \Delta t m \sigma_{x}+i \frac{\Delta t}{\Delta x} \sigma_{x}\right) \varphi(x-\Delta x, t) \\
= & P \varphi(x+\Delta x, t)+Q \varphi(x-\Delta x, t)
\end{aligned}
$$

with

$$
\begin{aligned}
P & \equiv \frac{1}{2}\left(-i \Delta t m \sigma_{x}-i \frac{\Delta t}{\Delta x} \sigma_{x}\right), \\
Q & \equiv \frac{1}{2}\left(1-i \Delta t m \sigma_{x}+i \frac{\Delta t}{\Delta x} \sigma_{x}\right) .
\end{aligned}
$$

Here, the one-step operator $C \equiv P+Q$ is defined and $\varphi(x, t)$ is not normalized in general. Therefore, when the wavefunction $\varphi(x, t)$ is normalized to $\tilde{\varphi}(x, t)$, we define a new one-step operator $\tilde{C}=\tilde{P}+\tilde{Q}$ to satisfy

$$
\varphi(x, t+\Delta t) \simeq \tilde{P} \tilde{\varphi}(x+\Delta x, t)+\tilde{Q} \tilde{\varphi}(x-\Delta x, t) .
$$

From Eq. (3.4), the quantum coin can be evaluated as

$$
\tilde{C}=\alpha\left(1-i \Delta t m \sigma_{x}\right)
$$

with the complex constant $\alpha$. To satisfy the unitary operator $\tilde{C}$, the parameter $\alpha$ should be set as

$$
\alpha=\frac{e^{i \theta}}{\sqrt{1+(\Delta t m)^{2}}}
$$

with the arbitrary real parameter $\theta$. Upon setting $m=1$, this can be reduced to the specific quantum coin in (2.6). Therefore, the discretized structure of the massive Dirac equation can simulate the one-dimensional DTQW. It is remarked that the massless Dirac equation can simulate the trivial DTQW. Note that similar approaches are well known as Dirac quantum cellular automata [44-47]. They have also exhibited various quantum dynamics by the massive Dirac equation.

This shows that the dynamical properties of a Dirac fermion is almost same as those of the DTQW, such as ballistic transport and the inverted-bell shape probability distribution. From Theorem 2.1, the Dirac fermion and DTQW have a one-to-one correspondence. Therefore, this result provides several experimental proposals in spintronics systems. Since spintronics systems have quantum-transport properties, this connection may reveal an efficient transport system. The Dirac particles in graphene and topological insulators are candidates for physical implementation of the DTQW. Since a topological insulator can be characterized by a topological phase, this must be one of the DTQW applications because the one-dimensional topological phase can be experimentally realized by a photonic implementation of the DTQW [17].

\section{Conclusion}

From the massive Dirac equation, we can derive the DTQWs with a specific coin. A spintronics system naturally has DTQW properties. However, higher-dimensional cases still remain unsolved - in particular, the correspondence to the chirality of the massive Dirac equation for more than three dimensions, whereas the one-dimensional case has been analyzed. The connection to the Majorana system has been discussed $[48,49]$. Furthermore, a DTQW with an arbitrary coin has been not yet been shown by controlling the time-dependent potential. For experimental implementation, the decoherence process [50-55] should be considered, although there has been no systematic methodology for a spintronics system.

\section{REFERENCES}

[1] Childs, A., Gosset, D., and Webb, Z., “Universal computation by multiparticle quantum walk," Science, 339: 791-794 (2013).

[2] Venegas-Andraca, S. E., Quantum Walks for Computer Scientists, Morgan and Claypool (2008).

[3] Konno, N., Quantum Potential Theory, Lecture Notes in Mathematics, Vol. 1954, Springer-Verlag (2008), p. 309.

[4] Venegas-Andraca, S. E., "Quantum walks: A comprehensive review," Quant. Inf. Proc., 11: 1015-1106 (2012).

[5] Kitagawa, T., "Topological phenomena in quantum walks: Elementary introduction to the physics of topological phases," Quant. Inf. Proc., 11: 1107-1148 (2012).

[6] Shikano, Y., "From discrete time quantum walk to continuous time quantum walk in limit distribution," J. Comput. Theor. Nanosci., 10: 1558-1570 (2013).

[7] Portugal, R., Quantum Walks and Search Algorithms, Springer (2013).

[8] Manouchehri, K., and Wang, J., Physical Implementation of Quantum Walks, Springer (2014).

[9] Feynman, R. P., "Space-time approach to non-relativistic quantum mechanics," Rev. Mod. Phys., 20: $367-387$ (1948).

[10] Gudder, S. P., Quantum Probability, Academic Press Inc. (1988).

[11] Aharonov, Y., Davidovich, L., and Zagury, N., "Quantum random walks," Phys. Rev. A, 48: 1687-1690 (1993).

[12] Meyer, D. A., "From quantum cellular automata to quantum lattice gases," J. Stat. Phys., 85: 551-574 (1996).

[13] Karski, M., Förster, L., Choi, J.-M., Steffen, A., Alt, W., Meschede, D., and Widera, A., "Quantum walk in position space with 
single optically trapped atoms," Science, 325: 174-177 (2009).

[14] Peruzzo, A., Lobino, M., Matthews, J. C. F., Matsuda, N., Politi, A., Poulios, K., Zhou, X.-Q., Lahini, Y., Ismail, N., Wörhoff, K., Bromberg, Y., Silberberg, Y., Thompson, M. G., and O’Brien, J. L., "Quantum walks of correlated photons," Science, 329 : 1500-1503 (2010).

[15] Schreiber, A., Cassemiro, K. N., Potoček, V., Gábris, A., Mosley, P. J., Andersson, E., Jex, I., and Silberhorn, Ch., "Photons walking the line: A quantum walk with adjustable coin operations," Phys. Rev. Lett., 104: 050502 (2010).

[16] Zähringer, F., Kirchmair, G., Gerritsma, R., Solano, E., Blatt, R., and Roos, C. F., "Realization of a Quantum Walk with One and Two Trapped Ions," Phys. Rev. Lett., 104: 100503 (2010).

[17] Kitagawa, T., Broome, M. A., Fedrizzi, A., Rudner, M. S., Berg, E., Kassal, I., Aspuru-Guzik, A., Demler, E., and White, A. G., "Observation of topologically protected bound states in photonic quantum walks," Nat. Commun., 3: 882 (2012).

[18] Schreiber, A., Gábris, A., Rohde, P. P., Laiho, K., Štefaňák, M., Potoček, V., Hamilton, C., Jex, I., and Silberhorn, Ch., "A 2D quantum walk simulation of two-particle dynamics," Science, 336: 55-58 (2012).

[19] Crespi, A., Osellame, R., Ramponi, R., Giovannetti, V., Fazio, R., Sansoni, L., De Nicola, F., Sciarrino, F., and Mataloni, P., "Anderson localization of entangled photons in an integrated quantum walk," Nat. Photon., 7: 322-328 (2013).

[20] Jeong, Y.-C., Di Franco, C., Lim, H.-T., Kim, M. S., and Kim, Y.-H., "Experimental realization of a delayed-choice quantum walk," Nat. Commun., 4: 2471 (2013).

[21] Fukuhara, T., Schauß, P., Endres, M., Hild, S., Cheneau, M., Bloch, I., and Gross, C., "Microscopic observation of magnon bound states and their dynamics," Nature, 502: 76-79 (2013).

[22] Xue, P., Qin, H., Tang, B., and Sanders, B. C., "Observation of quasiperiodic dynamics in a one-dimensional quantum walk of single photons in space," New J. Phys., 16: 053009 (2014).

[23] Travaglione, B. C., and Milburn, G. J., "Implementing the quantum random walk," Phys. Rev. A, 65: 032310 (2002).

[24] Sanders, B. C., Bartlett, S. D., Tregenna, B., and Knight, P. L., "Quantum quincunx in cavity quantum electrodynamics," Phys. Rev. A, 67: 042305 (2003).

[25] Lehman, L., Zatloukal, V., Brennen, G. K., Pachos, J. K., and Wang, Z., "Quantum walks with non-Abelian anyons," Phys. Rev. Lett., 106: 230404 (2011).

[26] Chandrashekar, C. M., "Disordered-quantum-walk-induced localization of a Bose-Einstein condensate," Phys. Rev. A, 83: 022320 (2011).

[27] Goyal, S. K., Roux, F. S., Forbes, A., and Konrad, T., "Implementing quantum walks using orbital angular momentum of classical light," Phys. Rev. Lett., 110: 263602 (2013).

[28] Hardal, A. Ü. C., Xue, P., Shikano, Y., Müstecaplıõlu, Ö. E., and Sanders, B. C., "Discrete-time quantum walk with nitrogenvacancy centers in diamond coupled to a superconducting flux qubit," Phys. Rev. A, 88: 022303 (2013).

[29] Moqadam, J. K., Portugal, R., and de Oliveira, M. C., "Quantum walks on a circle with optomechanical systems," Quantum Inf. Proc., 14: 3595-3611 (2015).

[30] Bracken, A. J., Ellinas, D., and Smyrnakis, I., "Free-Dirac-particle evolution as a quantum random walk," Phys. Rev. A, 75: 022322 (2007).

[31] Strauch, F. W., "Relativistic effects and rigorous limits for discrete- and continuous-time quantum walks," J. Math. Phys., 48: 082102 (2007).

[32] Sato, F., and Katori, M., "Dirac equation with an ultraviolet cutoff and a quantum walk," Phys. Rev. A, 81: 012314 (2010).

[33] Chandrashekar, C. M., Banerjee, S., and Srikanth, R., "Relationship between quantum walks and relativistic quantum mechanics," Phys. Rev. A, 81: 062340 (2010).

[34] Di Molfetta, G., Honter, L., Luo, B. B., Wada, T., and Shikano, Y., "Massless Dirac equation from Fibonacci discrete-time quantum walk," Quant. Stud.: Math. Found., 2: 243-252 (2015).

[35] Chisaki, K., Konno, N., Segawa, E., and Shikano, Y., "Crossovers induced by discrete-time quantum walks," Quant. Inf. Comp., 11: 741-760 (2011).

[36] Childs, A. M., "On the relationship between continuous- and discrete-time quantum walk," Commun. Math. Phys., 294: 581603 (2010).

[37] Di Molfetta, G., and Debbasch, F., "Discrete-time quantum walks: Continuous limit and symmetries," J. Math. Phys., 53: 123302 (2012).

[38] Knight, P. L., Roldán, E., and Sipe, J. E., "Propagating quantum walks: The origin of interference structures," J. Mod. Opt., 51: 1761-1777 (2004).

[39] de Valcárcel, G. J., Roldán, E., and Romanelli, A., "Tailoring discrete quantum walk dynamics via extended initial conditions," New J. Phys., 12: 123022 (2010).

[40] Shikano, Y., Wada, T., and Horikawa, J., "Discrete-time quantum walk with feed-forward quantum coin," Sci. Rep., 4: 4427 (2014).

[41] Ambainis, A., Bach, E., Nayak, A., Vishwanath, A., and Watrous, J., "One-dimensional quantum walks," in Proceedings of the 33rd Annual ACM Symposium on Theory of Computing (STOC'01) (ACM Press, New York, 2001), pp. 37-49.

[42] Konno, N., "Quantum random walks in one dimension," Quantum Inf. Proc., 1: 345-354 (2002).

[43] Konno, N., "A new type of limit theorems for the one-dimensional quantum random walk," J. Math. Soc. Jpn., 57: 1179-1195 (2005).

[44] Bialynicki-Birula, I., "Weyl, Dirac, and Maxwell equations on a lattice as unitary cellular automata," Phys. Rev. D, 49: 6920 (1994).

[45] D'Ariano, G. M., and Perinotti, P., "Derivation of the Dirac equation from principles of information processing," Phys. Rev. A, 90: 062106 (2014).

[46] Arrighi, P., Nesme, V., and Forets, M., "The Dirac equation as a quantum walk: Higher dimensions, observational convergence," J. Phys. A, 47: 465302 (2014). 
[47] Pérez, A., "Asymptotic properties of the Dirac quantum cellular automaton," Phys. Rev. A, 93: 012328 (2016).

[48] Asbóth, J. K., and Obuse, H., "Bulk-boundary correspondence for chiral symmetric quantum walks," Phys. Rev. B, 88: 121406(R) (2013).

[49] Asbóth, J. K., Tarasinski, B., and Delplace, P., "Chiral symmetry and bulk-boundary correspondence in periodically driven one-dimensional systems," Phys. Rev. B, 90: 125143 (2014).

[50] Kempe, J., "Quantum random walks: An introductory overview," Contemp. Phys., 44: 307-327 (2003).

[51] Kendon, V., "Decoherence in quantum walks - a review," Math. Struct. Comp. Sci., 17: 1169-1220 (2007).

[52] Liu, C., and Petulante, N., "One-dimensional quantum random walks with two entangled coins," Phys. Rev. A, 79: 032312 (2009).

[53] Gönülol, M., Aydıner, E., Shikano, Y., and Müstecaplıgõlu, Ö. E., "Survival probability in a one-dimensional quantum walk on a trapped lattice," New J. Phys., 13: 033037 (2011).

[54] Shikano, Y., "Differences between quantum walks and classical random walks in limit distributions," AIP Conf. Proc., 1327: 487 (2011).

[55] Shikano, Y., Horikawa, J., and Wada, T., "The discrete-time quantum walk as a stochastic process in quantum mechanics," Phys. Scr., T151: 014016 (2012). 